\title{
Results of experimental studies and numerical modeling of multistage waveguide-slotted membranes filters with complex slots geometry
}

\author{
Natalia Kopylova ${ }^{1, *}$, Alexei Kopylov ${ }^{1}$, and Yuri Salomatov ${ }^{1}$ \\ ${ }^{1}$ Siberian Federal University, 660079 Krasnoyarsk, Russia
}

\begin{abstract}
The article contains the results of an experimental study and numerical simulation of the amplitude-frequency characteristics of one, three and four-stage filters on waveguide-slotted membranes with complex resonance slot geometry membranes located in a rectangular $35 \times 15 \mathrm{~mm}$ waveguide. The design of filters and the topology of a complex slot in the membrane are shown. The results can be useful in the design of waveguide filters in the frequency range $5.5 \ldots 8.5 \mathrm{GHz}$.
\end{abstract}

\section{Introduction}

As is known [1], carrying in regular rectangular waveguide inhomogeneity of one or another type is the one of the main ideas for realization microwave filters based on rectangular waveguides. In largest number cases, the reactive pins located along the axis of symmetry of the wide wall of the waveguide in the direction of propagation of the microwave electromagnetic wave are used as inhomogeneities. The most significant drawback of such filters, in our opinion, is their relatively large dimensions, since the distances between reactive pins should be commensurate with a quarter of the microwave wavelength at operating frequencies.

As was shown earlier [2], one of the possible ways to reduce the dimensions of waveguide filters is the formation of band-pass and band-stopping amplitude-frequency characteristics (AFC) of these filters by using thin metal membranes in their structure having resonant slots of various shapes (topology, geometry). In particular, the geometry of such slots varies from simple linear shapes [2] to angular ones located at different points in the cross-section of a rectangular waveguide [3].

Varying the geometry and location of the resonant slots allows one to achieve an increase in selectivity and obtain an AFC of a certain form. In addition, in order to increase the selectivity in such filters, a well-known cascading technique is used $[2,3]$. In this case slotted membranes are cascaded with waveguide inserts of different working cross-sections and thicknesses.

In this paper we present the results of an experimental study and numerical simulation of three filters on waveguide-slotted membranes (WSM) having complex shape located in a rectangular waveguide with a working cross-section of $35 \times 15 \mathrm{~mm}$.

* Corresponding author: kopaph@yandex.ru 


\section{Filters design}

The structures of the first, second and third filters are shown in Figure 1a), b), c), respectively.

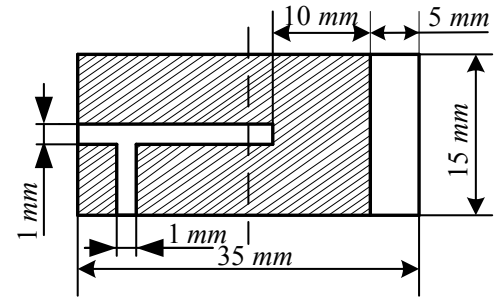

a)
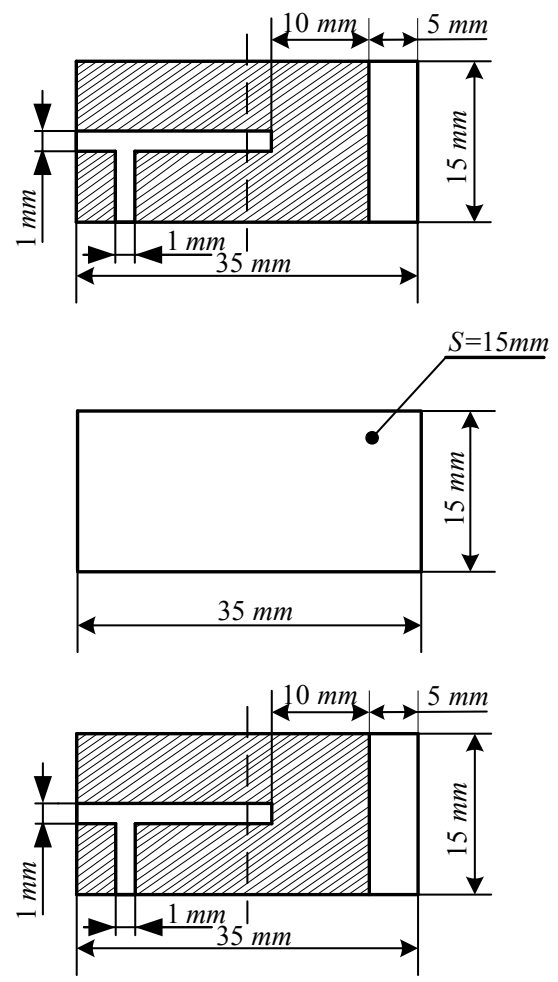

b)
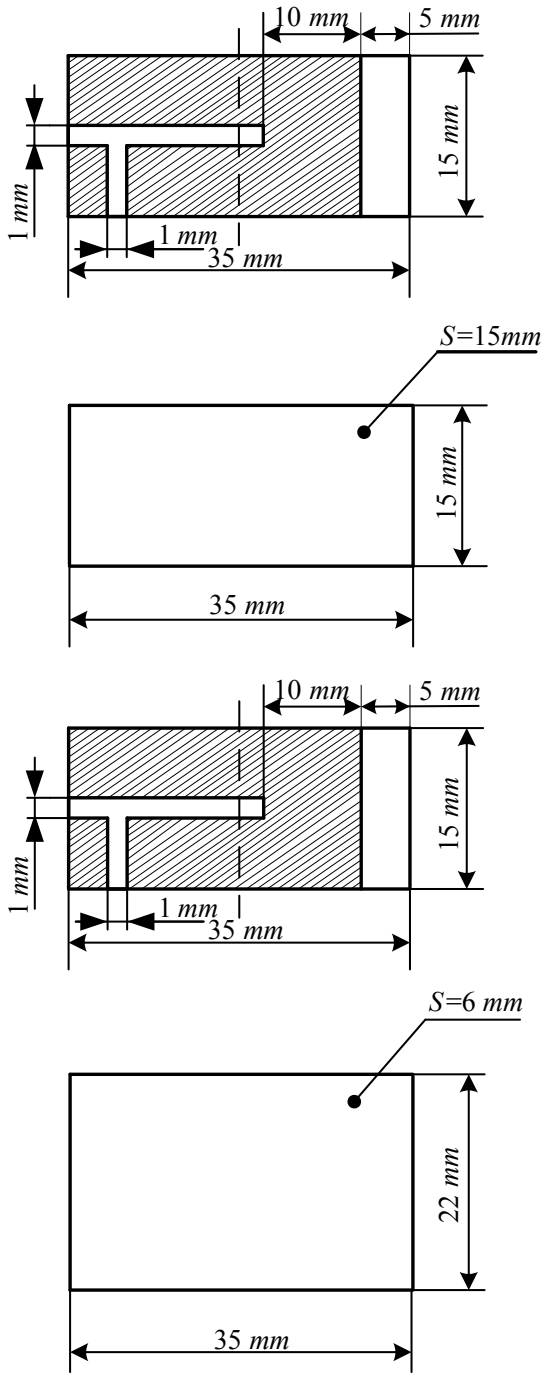

c)

Fig. 1. The structure of the filters: a) the first filter, b) the second filter, c) the third filter

The first filter contains a single membrane with a complex geometry slot. This slot membrane is clamped between input and output waveguide-coaxial transitions (WCT) and is located perpendicular to the propagation direction of the microwave wave. The thickness of the membrane is $0.25 \mathrm{~mm}$.

The second filter is a three-stage system and consists of a first single slotted cascade, a waveguide spacer $\mathrm{S}=15 \mathrm{~mm}$ thick with a cross-section of $35 \times 15 \mathrm{~mm}$ and a second single slotted membrane. The geometries of the slots at the slot membranes of this filter are 
similar to the geometry of the slots at the slot membrane of the first filter. The second slot membrane positioned symmetrically to the first membrane.

The third filter is a four-stage and consists of a first single slotted cascade, first waveguide spacer $\mathrm{S}=15 \mathrm{~mm}$ thick with a working cross-section of $35 \times 15 \mathrm{~mm}$, a second single slotted membrane with a slot geometry similar to the geometry of the first membrane and arranged symmetrically to the location of the first and second waveguide spacers $S=6$ $\mathrm{mm}$ thick with a working section of $35 \times 22 \mathrm{~mm}$.

The second and third filters, like the first filter, are clamped between input and output WCT for experimental studies and are perpendicular to the direction of propagation of electromagnetic waves in the waveguide.

The topology of the slotted membranes was chosen experimentally to obtain AFC filters closest to rectangular band-pass characteristics in the range $6 \ldots 7 \mathrm{GHz}$. Designs of the second and third filters with waveguide inserts are chosen experimentally.

\section{Results and Discussions}

Measurements of the frequency response of the filters were carried out on a scalar attenuation meter (voltage transmission coefficients, or voltage gain $\left|\mathrm{K}_{\mathrm{U}}\right|$ ) and VSWR in the manual mode of frequency tuning by points. The Figure 2, Figure 3, Figure 4 shows the results of measurements of the values of the transmission coefficients for voltage $\left|\mathrm{K}_{U}\right|$ in $\mathrm{dB}$ by voltage (vertical axis of the figures) against the corresponding frequencies (horizontal axis of the drawings) for the first, second and third filters, respectively. The experimental values are denoted by circular points that are connected by a solid curve.

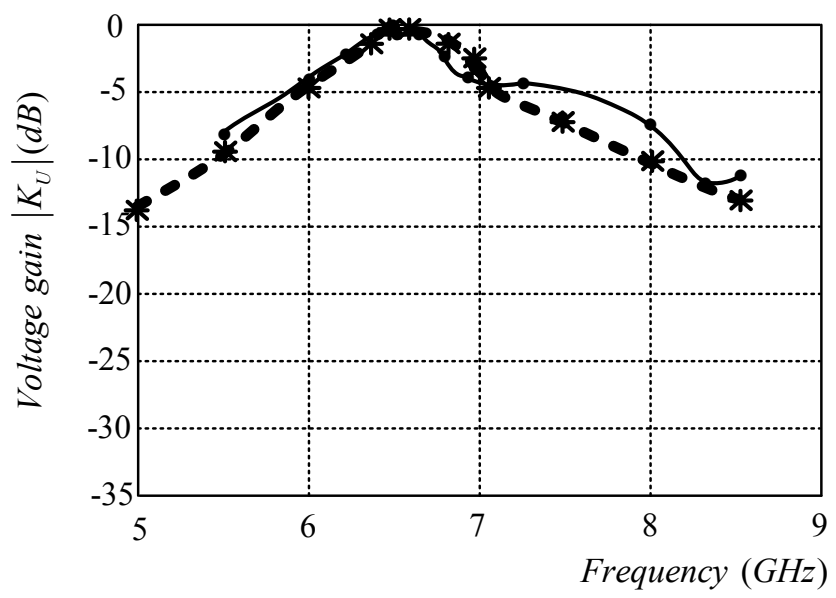

Fig. 2. Amplitude-frequency characteristics of first filter

For the same three filters was made simulation AFC by known numerical methods of electrodynamics. Results of this simulation in the form of asterisks are plotted in the same Figure2, Figure 3, Figure 4 corresponding to the first, second and third filters; the asterisks in each figure are connected by a dashed curve.

Analysis of the obtained experimental frequency dependences of $\left|\mathrm{K}_{\mathrm{U}}\right|$ for all three filters shows that response these filters are passband by the type of frequency. At the same time, the frequency-selective properties of the first filter containing only one slot membrane providing attenuation beyond the pass band to $12 \mathrm{~dB}$ at a frequency of about $8.35 \mathrm{GHz}$ and $8 \mathrm{~dB}$ at a frequency of $5.5 \mathrm{GHz}$. It seems to us satisfactory given the insignificant dimensions of the filter itself on such a single membrane - the dimensions of the filter are 
almost equal to the dimensions of the two WCR - input and output. The minimum losses of the first filter were $0.45 \ldots 0.8 \mathrm{~dB}$ in the pass band $6.4 \ldots 6.7 \mathrm{GHz}$.

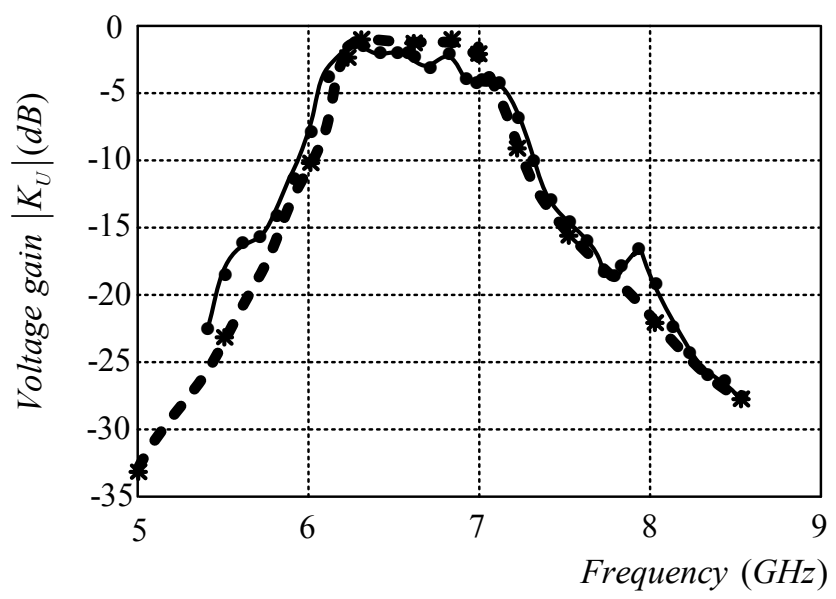

Fig. 3. Amplitude-frequency characteristics of second filter

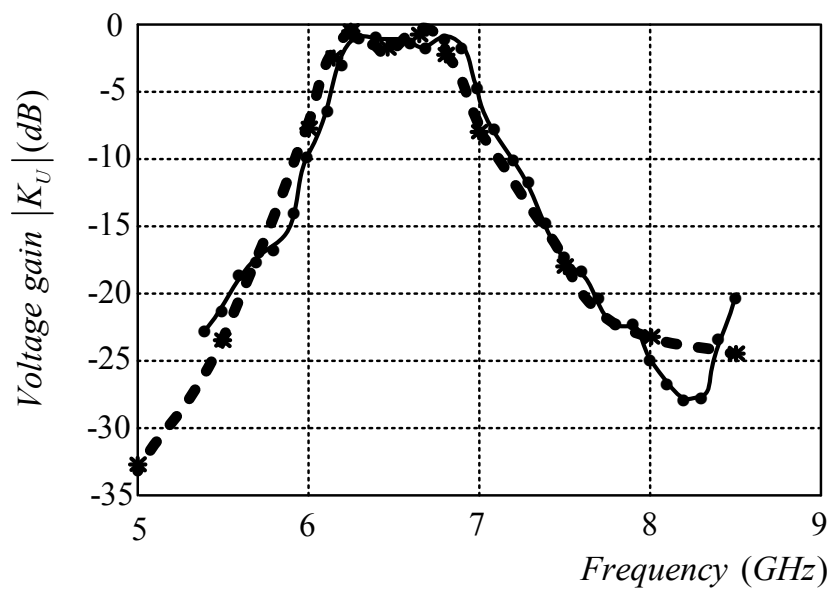

Fig. 4. Amplitude-frequency characteristics of third filter

With an increase of number filter stages, the attenuation beyond the pass-band also increases, the bandwidth of the transmitted frequencies decreases, and the slopes of the amplitude-frequency characteristics increase. So, for the second, three-stage filter, the attenuation beyond the pass-band reaches a value of $23 \mathrm{~dB}$ at a frequency of $5.5 \mathrm{GHz}, 27$ $\mathrm{dB}$ at a frequency of $8.5 \mathrm{GHz}$. At the same time, the minimum losses increase to $0.8 \ldots 1.8$ $\mathrm{dB}$ in the $6.2 \ldots 6.6 \mathrm{GHz}$ band. We can see, the AFC of the second filter shifts in frequency to the region of lower frequencies with respect to the AFC of the first filter by about 0.2 GHz. A visual comparison of the AFC of the second filter with the frequency response of the first filter shows a sharp increase in the steepness of the slopes of the amplitudefrequency characteristic of the three- stages filter with respect to the single- stages filter.

For the third, four- stages filter, attenuation beyond the pass-band reaches $23 \mathrm{~dB}$ at a frequency of $5.5 \mathrm{GHz}, 27 \mathrm{~dB}$ at $8.2 \ldots 8.3 \mathrm{GHz}$ with a minimum loss of $0.65 \ldots 1.4 \mathrm{~dB}$ in the frequency band $6.25 \ldots 6.85 \mathrm{GHz}$. Apparently, the AFC of the third filter is practically 
not shifted in frequency to the region of lower frequencies with respect to the second filter AFC. Moreover, the frequency bandwidth of the third filter has become larger than the bandwidth of the second filter at the same level of loss in this band. Visually, there is a noticeable increase in the steepness of the slopes of the amplitude-frequency response of the four-link filter relative to the three- stages filter. It should be noted that in the frequency response of the third filter there is a feature regarding the frequency response of the first and second filters - there is a sharp decline of AFC curve at $8.2 \ldots 8.3 \mathrm{GHz}$ to value of attenuation about $28 \mathrm{~dB}$ with followed sharp increase of AFC curve at $8.5 \mathrm{GHz}$ to value of attenuation about $20 \mathrm{~dB}$. We believe that this is evidence of the next pass-band appearance.

\section{Conclusions}

The greatest differences between the values obtained experimentally and by numerical simulation are observed in the parameter of the lowest attenuation value introduced by the filters in the pass-band. The experimental values of the losses introduced by the filters in the pass-band are $0.45 \ldots 1.8 \mathrm{~dB}$ for the filters we studied, whereas the calculated values of this parameter are $0.05 \ldots 0.5 \mathrm{~dB}$. In the parameter of the insertion loss value introduced by the filters behind the pass-bands, in the stop-bands, there is a practical equality of the numerical values between the experimental and the calculated values. The experimental values of the insertion loss by the filters in the stop bands amount to $23 \ldots 27 \mathrm{~dB}$ for the three-stage and four-stage filters, and they are practically equal to the calculated values.

This behavior of these parameters is explained by the presence of losses, the values of which are not taken into account at the moment in our modeling. The presence of additional losses just leads to a noticeable increase in the losses in the pass bands and to a certain decrease in the attenuation values in the stop bands, which become noticeable only at very large attenuations of the order of $50 \ldots 70 \mathrm{~dB}$ or more.

The carried out researches show that qualitatively the frequency response obtained by the experimental and calculated paths coincides almost completely and has some insignificant quantitative differences. The obtained results allow speaking about the possibility of confident modeling of WSM-structures for the purpose of creating efficient microwave filtering devices.

\section{References}

1. R. V. Snyder, A. Mortazawi, I. Hunter, S. Bastioli, G. Macchiarella, K. Wu, IEEE Trans. on MTT 63, 10, (2015)

2. C. A. Leal-Sevillano, J. R. Montejo-Garai, J., A. Ruiz-Cruz and J. M. Rebollar, IEEE Trans. on MTT 64, 3, (2016)

3. A. Kopylov, LAP LAMBERT Academic Publishing; ISBN: 978-3-659-67685-7, (2015) 